\title{
Pharmacokinetic and Pharmacodynamic Modelling to Characterize the Tolerability of Alternative Up-Titration Regimens of Roflumilast in Patients with Chronic Obstructive Pulmonary Disease
}

\author{
Axel Facius $^{1} \cdot$ Eleonora Marostica $^{2} \cdot$ Philip Gardiner $^{3} \cdot$ Henrik Watz $^{4} \cdot$ Gezim Lahu $^{1}$
}

Published online: 25 May 2018

(C) The Author(s) 2018

\begin{abstract}
Background In the OPTIMIZE study, 4 weeks of roflumilast $250 \mu \mathrm{g}$ once daily before escalation to the approved $500 \mu \mathrm{g}$ once daily maintenance dose reduced treatment discontinuations and improved tolerability to roflumilast among patients with chronic obstructive pulmonary disease (COPD). In this study, we present the pharmacokinetic (PK) results and PK/pharmacodynamic (PD) modelling data from OPTIMIZE.

Methods OPTIMIZE was a multicentre, double-blind, phase III study in which patients with severe COPD were randomized 1:1:1 to receive oral roflumilast $250 \mu \mathrm{g}$ once daily, $500 \mu \mathrm{g}$ every other day, or $500 \mu \mathrm{g}$ once daily for 4 weeks, followed by $500 \mu \mathrm{g}$ once daily for 8 weeks. A population PK (popPK) model characterized roflumilast exposure levels (total phosphodiesterase-4 inhibition [tPDE4i]). Furthermore, models characterized the percentage of patients with adverse events (AEs) of interest
\end{abstract}

Electronic supplementary material The online version of this article (https://doi.org/10.1007/s40262-018-0671-4) contains supplementary material, which is available to authorized users.

Axel Facius

axel.facius@thinkq2.com

1 thinkQ2 AG, Oberneuhofstr. 5, 6340 Baar, Switzerland

2 LAP\&P Consultants BV, Archimedesweg 31, 2333 CM Leiden, The Netherlands

3 RIA QCP, IMED Biotech Unit, AstraZeneca, Gothenburg, Sweden

4 Airway Research Center North (ARCN), Member of the German Center for Lung Research (DZL), Pulmonary Research Institute at Lung Clinic Grosshansdorf, Grosshansdorf, Germany
(PK/AE model), and time to discontinuation due to such AEs (PK/time-to-event model).

Results The popPK model adequately described average plasma concentrations and variability from 1238 patients. The percentage of patients with AEs of interest increased with predicted tPDE4i exposure (logit scale slope 0.484; confidence interval 0.262-0.706; $p=2 \times 10^{-5}$ ). PK/timeto-event model analysis predicted that patients receiving the $250 \mu \mathrm{g}$ up-titration regimen had significantly lower discontinuation rates and longer time to discontinuation compared with roflumilast $500 \mu \mathrm{g}$ every other day or $500 \mu \mathrm{g}$ once daily $(p=0.0014)$.

Conclusions In this PK/PD model, a 4-week up-titration regimen with roflumilast $250 \mu \mathrm{g}$ once daily was found to reduce discontinuations and improve tolerability, confirming the main clinical findings of the OPTIMIZE study. However, use of this lower dose as long-term maintenance therapy may not induce sufficient phosphodiesterase-4 inhibition to exert clinical efficacy, supporting the approval of $500 \mu \mathrm{g}$ as maintenance dose.

Trial Registration OPTIMIZE: NCT02165826; REACT: NCT01329029. 


\section{Key Points}

The oral phosphodiesterase-4 inhibitor roflumilast reduces exacerbations of chronic obstructive pulmonary disease, but its use is associated with tolerability issues in some patients during the first few weeks of therapy.

Alternative dosing regimens have been investigated in the OPTIMIZE study. Starting roflumilast at a lower dose ( $250 \mu \mathrm{g}$ daily) for 4 weeks, before escalation to the $500 \mu \mathrm{g}$ maintenance dose, was shown to improve tolerability and reduce discontinuations.

Modelling data support this up-titration regimen as a means of reducing the risk of adverse events and early treatment discontinuations, enabling patients to stay on roflumilast treatment for longer at the maintenance dose.

\section{Introduction}

Chronic obstructive pulmonary disease (COPD) is characterized by persistent respiratory symptoms and airflow limitation due to airway and/or alveolar abnormalities, and is usually caused by significant exposure to noxious particles or gases [1]. Chronic inflammation plays a key role in COPD [2]. Frequent COPD exacerbations accelerate disease progression and increase the risk of mortality [3]. Despite recommended inhaled therapies, many patients with severe COPD continue to experience exacerbations [4].

Roflumilast is a selective, oral phosphodiesterase-4 (PDE4) inhibitor used for the treatment of patients with severe COPD associated with chronic bronchitis and a history of exacerbations [5]. Clinical studies have shown that roflumilast, taken once daily as add-on to inhaled COPD therapy, reduces exacerbations in this patient population [6,7]. Most recently, this has been shown in the REACT study in patients using roflumilast therapy on top of an inhaled corticosteroid (ICS)/long-acting beta agonist $(\mathrm{LABA}) \pm$ long-acting muscarinic antagonist (LAMA) combination [8].

Roflumilast is rapidly metabolized to its only active metabolite, roflumilast $\mathrm{N}$-oxide, which has specificity and potency similar to roflumilast [11], and is estimated to account for approximately $90 \%$ of the total PDE4 inhibition (tPDE4i), with $10 \%$ attributed to the parent roflumilast. Steady state plasma concentrations, achieved after 3-4 days of once daily dosing, can be used to estimate the combined PDE4i of roflumilast and roflumilast $\mathrm{N}$-oxide
[12]. The PK of roflumilast are linearly dose-proportional over $250-1000 \mu \mathrm{g}$, and it is rapidly and almost completely absorbed, with a mean absolute bioavailability of approximately $80 \%$ after a single $500 \mu \mathrm{g}$ dose [13]. The maximum concentration $\left(C_{\max }\right)$ of roflumilast is reached within $1 \mathrm{~h}, 4-8 \mathrm{~h}$ with roflumilast $\mathrm{N}$-oxide, and remains constant for $6-8 \mathrm{~h}$. Steady-state plasma concentrations of roflumilast and roflumilast $\mathrm{N}$-oxide are achieved within 4 and 6 days, respectively [13]. The effective half-life of roflumilast ranges from 8 to $31 \mathrm{~h}$, with a median of $17 \mathrm{~h}$, and the half-life of roflumilast $\mathrm{N}$-oxide is approximately $30 \mathrm{~h} \mathrm{[13].}$

Side effects most commonly reported with roflumilast include diarrhoea, nausea and a reduction in body weight, and, while typically mild-to-moderate in severity, they are a common cause of early treatment discontinuation $[9,10]$. However, the transient nature of the gastrointestinal side effects indicates that patients may develop tolerance with continued dosing [10], and alternative dosing strategies during the first few weeks of treatment may help reduce treatment discontinuations.

A population pharmacokinetic (popPK) model developed using data from 28 phase I-III studies demonstrated that tPDE4i can be used as a predictor of roflumilast tolerability [12]. This model also predicted that side effects may be reduced with alternative roflumilast dosing using $250 \mu \mathrm{g}$ once daily, or $500 \mu \mathrm{g}$ every other day [14]. These dosing strategies were investigated further in the doubleblind, phase III OPTIMIZE study (NCT02165826), which found that up-titration from roflumilast $250 \mu \mathrm{g}$ once daily for 4 weeks to the approved maintenance dose of $500 \mu \mathrm{g}$ once daily improved tolerability and reduced treatment discontinuation, compared with starting at a dose of $500 \mu \mathrm{g}$ [15].

In this study, we report PK/pharmacodynamic (PD) analyses from the OPTIMIZE study. The previously validated popPK model [12], enhanced with data from the REACT [8] and OPTIMIZE studies [15], was used to further evaluate the exposure-tolerability relationship of the alternative roflumilast dosing regimens.

\section{Methods}

\subsection{Study Design}

A non-linear, mixed-effect, integrated popPK model was employed with covariate-effect and random-effect parameters that were estimated using the combined dataset from the OPTIMIZE and REACT studies.

The methodology for both OPTIMIZE and REACT have been described previously $[8,15]$. In brief, OPTIMIZE was a randomized, double-blind, three-arm, parallel- 
group, phase III clinical trial in patients with COPD (1321 patients), which evaluated a 4-week up-titration regimen of roflumilast at treatment initiation before escalating to the approved $500 \mu \mathrm{g}$ once daily maintenance dose. Patients were randomized (1:1:1) to 4 weeks (double-blind) of oral roflumilast $250 \mu \mathrm{g}$ once daily, $500 \mu \mathrm{g}$ every other day, or $500 \mu \mathrm{g}$ once daily, all followed by roflumilast $500 \mu \mathrm{g}$ once daily for 8 weeks (single-blind) [15]. The OPTIMIZE study also included an open-label down-titration period to evaluate the tolerability and PK of roflumilast $250 \mu \mathrm{g}$ once daily in patients who had dropped out of the main study [15]. REACT (NCT01329029) was a randomized, double-blind, parallel-group, multicentre, phase III clinical trial in patients with severe to very severe COPD (1945 patients). Patients were randomized (1:1) to receive either roflumilast $500 \mu \mathrm{g}$ once daily or placebo for 52 weeks [8]. In both studies, roflumilast was administered as a yellow film-coated tablet (EU formulation).

Both studies included patients $\geq 40$ years of age with COPD associated with chronic productive cough and a history of exacerbations in the previous 12 months (one or more prior exacerbation in OPTIMIZE; two or more exacerbations in REACT), who were current or former smokers, and had a post-bronchodilator forced expiratory volume in $1 \mathrm{~s}\left(\mathrm{FEV}_{1}\right) \leq 50 \%$ of predicted, and $\mathrm{FEV}_{1} /$ forced vital capacity (FVC) ratio $<70 \%$. In OPTIMIZE, patients received study medication on top of their usual standard-of-care COPD maintenance treatment, which could include LABA/ICS \pm LAMA and/or theophylline. In REACT, patients received study treatment on top of a background of ICS/LABA with or without LAMA [8].

Both studies were conducted in accordance with the Declaration of Helsinki, and the International Conference on Harmonisation (ICH), Good Clinical Practice and applicable local regulations. All patients provided written informed consent to participate.

\subsection{Objectives}

The objectives of the current study were to characterize the exposure of COPD patients to evaluate the exposure-tolerability relationship in both up-titration and down-titration scenarios.

\subsection{Bioanalytical Methods}

Across the phase I-III [12], REACT [8] and OPTIMIZE studies [15], roflumilast and roflumilast $\mathrm{N}$-oxide concentrations in plasma samples were quantified using validated high-performance liquid chromatographic systems coupled with tandem mass spectrometric detection.

\subsection{Model Development}

A sequential modelling approach was performed to develop the PK/PD models. The PK (exposure) were characterized first (independently of the PD adverse events [AEs]). The final integrated popPK model was used to predict individual exposure levels (average concentrations of roflumilast and roflumilast $\mathrm{N}$-oxide), which were in turn used to derive individual tPDE4 $\mathrm{i}$ activities for all three treatments $(250 \mu \mathrm{g}$ once daily $/ 500 \mu \mathrm{g}$ once daily, $500 \mu \mathrm{g}$ every other day $/ 500 \mu \mathrm{g}$ once daily, and $500 \mu \mathrm{g}$ once daily).

Individual tPDE4 $\mathrm{i}$ activity levels were then used to characterize the PK/tolerability relationship in terms of the percentage of patients with AEs (PK/AE model) and time to discontinuation due to AEs (PK/time-to-event model).

Please see supplementary Methods 1-4 in the online resource for further details of the Methods for the development of all models.

\subsubsection{Integrated Population Pharmacokinetic (popPK) Model}

The integrated popPK model was developed from an earlier base model in which the structural parameters were fixed to estimates obtained using a dataset of 21 phase I and two phase II/III studies [12]. This existing base model describes the PK of both roflumilast (parent) and roflumilast $\mathrm{N}$-oxide (metabolite) simultaneously. It consists of two distribution compartments, with first-order absorption and elimination for the parent, and two additional distribution compartments with first-order elimination for the metabolite (Fig. 1).

The existing base model was applied to the OPTIMIZE data only according to a Bayesian feedback procedure [16] (i.e. MAXEVAL $=0$ in the $\mathrm{NONMEM}^{\circledR}$ code [12] [estimation is not performed but parameters already available are used to get predictions for the new OPTIMIZE dataset]). This analysis showed that the base model

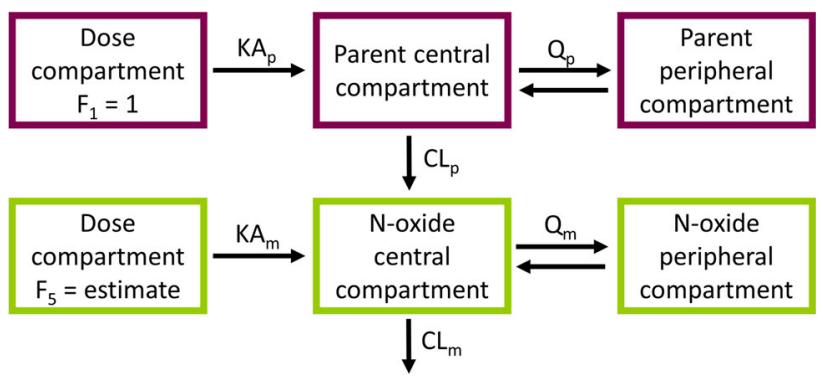

Fig. 1 PopPK model for roflumilast and roflumilast $\mathrm{N}$-oxide. Subscripts indicate parameters belonging to parent (p) or metabolite (m). $C L$ clearance, $K A$ absorption rate constant, $Q$ intercompartmental clearance, $F$ relative oral bioavailability, popPK population pharmacokinetic 
satisfactorily described the OPTIMIZE population and was used to estimate the phase II/III patient effects (i.e. dichotomous parameters describing significant differences in model parameters between healthy volunteers and patients); between-subject variability (BSV) and residual error, on the combined dataset (OPTIMIZE and REACT). The covariates included in the base model were re-estimated on the current combined dataset (OPTIMIZE and REACT). Finally, a formal covariate analysis was performed to assess whether additional covariates not included in the base model (i.e. age, sex, and race) had a statistically significant effect using the combined dataset.

\subsubsection{Pharmacokinetic/Adverse Event (PK/AE) and PK/Time-to-Event Model}

Analyses were performed in order to characterize the relationship of systemic exposure with the percentage of patients with at least one $\mathrm{AE}$ (PK/AE model), and the relationship of systemic exposure with time to treatment discontinuation due to AEs (PK/time-to-event model).

The tPDE4i values were tabulated and merged to the AE and time-to-event data to obtain the respective PK/AE and PK/time-to-event analysis datasets. AEs were coded according to the Medical Dictionary for Regulatory Activities (MedDRA) version 18, and assigned to preferred terms. Events were grouped into 'AEs of interest': headache, diarrhoea, nausea, vomiting, abdominal pain, appetite disorders, sleep disorders, angioedema, anxiety, depression and weight loss (online resource Table S3). Note that this definition of 'AEs of interest' is slightly broader than the definition used in the safety analysis of the OPTIMIZE study [15], for consistency with previous PK/ AE analyses [12].

A logistic regression model was used to characterize the relationship between tPDE4i and the frequency of patients with AEs (PK/AE model). The AE status was assumed to follow a binomial distribution and modelled using logistic regression:

$\log \operatorname{it}(p)=$ intercept $+t P D E 4 i \cdot$ slope $+\in \sim N\left(0, \sigma^{2}\right)$.

The variables sex, age, race, smoking status, body weight, COPD status, concomitant treatment with LAMA, statins, and LABA/ICS were tested as covariates on intercept and slope following a standard forward inclusion $(p<0.05)$, backward deletion $(p<0.01)$ procedure [17].

A parametric time-to-event model was used to characterize the time to treatment discontinuation due to AEs and other covariates during the initial 4-week up-titration phase (PK/time-to-event model). Parametric time-to-event models attempt to fit a given random distribution (for example, normal) to the distribution of observed event times by estimating distribution parameters such as position and shape. Using the normal distribution, position would be the mean and shape would be the variance. The variables tPDE4i, treatment arm, sex, age, race, smoking status, body weight, COPD status, concomitant treatment with LAMA, statins, and LABA/ICS were tested as covariates on position following a standard forward inclusion $(p<0.05)$, backward deletion $(p<0.01)$ procedure.

\subsection{Outcomes and Endpoints}

Endpoints were (1) the satisfactory prediction of roflumilast and roflumilast $\mathrm{N}$-oxide plasma levels, and hence tPDE4i activity (exposure), using the integrated popPK model; (2) the association of predicted exposure with the percentage of patients with AEs of interest using the PK/AE model; (3) the association of predicted exposure with the percentage of patients prematurely discontinuing study treatment using the PK/time-to-event model.

\section{Results}

\subsection{Patients}

Baseline characteristics of REACT and OPTIMIZE patients are shown in Table 1. The patient study flow for OPTIMIZE has been published previously [15]. In the OPTIMIZE study, 1323 patients were randomized; however, no plasma samples were available for 72 patients, and PK measurements were below the limit of detection for 13 patient samples. For the resulting 1238 patients, a total of 18,983 quantifiable plasma samples for PK measurements were available. This comprised 5878 samples $(250 \mu \mathrm{g}$ once daily/500 $\mu \mathrm{g}$ once daily), 5835 samples (500 $\mu \mathrm{g}$ every other day/500 $\mu \mathrm{g}$ once daily) and 5556 samples $(500 \mu \mathrm{g}$ once daily) in the trial, and 1714 samples $(250 \mu \mathrm{g}$ once daily) in the down-titration phase (online resource Table S1).

Of the 1945 randomized patients in the REACT study, plasma samples were available from 461 patients, of which 3176 were quantifiable. The demographics of patients enrolled in OPTIMIZE and REACT were well matched (mean age $64.5 \pm 8.1$ and $64.2 \pm 8.4$ years; 74.4 and $76.8 \%$ male; 46.6 and $47.7 \%$ current smokers, respectively). The resulting OPTIMIZE and REACT PK datasets were combined.

\subsection{Integrated PopPK Model}

The integrated popPK model was able to adequately describe total plasma concentrations of roflumilast and its metabolite, as well as the BSV across all treatment phases 
Table 1 Demographic and baseline characteristics for OPTIMIZE, REACT and combined datasets included in the model

\begin{tabular}{|c|c|c|c|}
\hline Characteristic & OPTIMIZE & $\begin{array}{l}\text { REACT } \\
(500 \mu \mathrm{g} \text { roflumilast group })\end{array}$ & Combined \\
\hline Patients $^{\mathrm{a}}$ & $1238(72.9)$ & $461(27.1)$ & $1699(100.0)$ \\
\hline $250 \mu \mathrm{g} \mathrm{OD} / 500 \mu \mathrm{g}$ OD & 409 & - & - \\
\hline $500 \mu \mathrm{g} \mathrm{EOD} / 500 \mu \mathrm{g}$ OD & 408 & - & - \\
\hline $500 \mu \mathrm{g}$ OD & 392 & - & - \\
\hline $250 \mu \mathrm{g}$ OD DT & 101 & - & - \\
\hline \multicolumn{4}{|l|}{ Samples ${ }^{\mathrm{a}}$} \\
\hline Roflumilast & $9416(85.6)$ & $1589(14.4)$ & $11,005(100.0)$ \\
\hline Roflumilast N-oxide & $9553(85.8)$ & $1587(14.2)$ & $11,140(100.0)$ \\
\hline \multicolumn{4}{|l|}{ Age, years } \\
\hline Mean (SD) & $64.5(8.1)$ & $64.2(8.4)$ & $64.4(8.2)$ \\
\hline Median (Min, Max) & $64.0(40,90)$ & $64.0(41,92)$ & $64.0(40,92)$ \\
\hline \multicolumn{4}{|l|}{$\operatorname{Sex}^{\mathrm{b}}$} \\
\hline Male & $921(74.4)$ & $354(76.8)$ & $1275(75.0)$ \\
\hline Female & $317(25.6)$ & $107(23.2)$ & $424(25.0)$ \\
\hline \multicolumn{4}{|l|}{ Smoking status ${ }^{\mathrm{b}}$} \\
\hline Current & $577(46.6)$ & $220(47.7)$ & $797(46.9)$ \\
\hline Former & $661(53.4)$ & $241(52.3)$ & $902(53.1)$ \\
\hline \multicolumn{4}{|l|}{ Race $^{\mathrm{b}}$} \\
\hline White & $1132(91.4)$ & $447(97.0)$ & $1579(92.9)$ \\
\hline Asian & $82(6.6)$ & $8(1.7)$ & $90(5.3)$ \\
\hline Black or African American & $9(0.7)$ & $4(0.9)$ & $13(0.8)$ \\
\hline Other & $9(0.7)$ & $2(0.4)$ & $11(0.6)$ \\
\hline Hispanic & $6(0.5)$ & $0(0.0)$ & $6(0.4)$ \\
\hline \multicolumn{4}{|l|}{ Weight, kg } \\
\hline Mean (SD) & $75.4(17.80)$ & $75.0(17.60)$ & 75.3 (17.74) \\
\hline Median (min, max) & $74.0(33.5,160)$ & $73.0(39,155)$ & $74.0(33.5,160)$ \\
\hline
\end{tabular}

Data are expressed as $n(\%)$ unless otherwise specified

$D T$ down-titration period, $E O D$ every other day, $O D$ once daily, $S D$ standard deviation, $\min$ minimum, $\max$ maximum

${ }^{a}$ Percentages relative to total number in the combined dataset

${ }^{\mathrm{b}}$ Percentages relative to total number in the study (up-titration, maintenance, and down-titration) and dosing schemes. This can be seen in the visual predictive checks (Fig. 2). Overall, parameters of the integrated popPK model (based on the combined REACT and OPTIMIZE dataset) were estimated with good precision (coefficient of variation $[\mathrm{CV}]<50 \%$ ), and parameter values were consistent with previous findings (online resource Table S2).

Figure 3 illustrates derived covariate effects on steadystate tPDE4i (red) compared with previously identified covariate effects in healthy volunteers (blue). While the model predicted some influence of age, sex, body weight, and smoking habits on exposure, the effects were considered not clinically relevant and did not warrant change to current approved maintenance dosing.

\subsection{PK/AE Model}

The PK/AE model is a logistic regression model used to characterize the relationship between tPDE4i and frequency of patients with AEs of interest. The final PK/AE model quantified a significant increase in the percentage of patients with AEs of interest with increased exposure (tPDE4i slope on the logit scale was 0.484 ; confidence interval [CI] $0.262-0.706 ; p=2 \times 10^{-5}$ ) (Fig. 4). In addition, the covariates 'concomitant LAMA' and 'smokers' indicated that percentages of patients with AEs of interest were higher in patients concomitantly treated with LAMAs when compared with patients not taking LAMAs (effect of concomitant LAMA on the logit scale: 0.354; 
Fig. 2 Visual predictive checks showing variability in roflumilast and roflumilast $\mathrm{N}$-oxide exposures. Visual predictive checks of $500 \mu \mathrm{g}$ OD exposures for each treatment arm for roflumilast (top panels) and roflumilast $\mathrm{N}$-oxide (bottom panels) for patients receiving a $500 \mu \mathrm{g}$ OD from all treatment arms, b $500 \mu \mathrm{g}$ EOD (uptitration arm 2), or c $250 \mu \mathrm{g}$ OD (up-titration arm 3). Purple line and grey area represent the median prediction and $90 \%$ green and red dotted lines represent median observation and 5th and 95th percentiles of observations, respectively; grey dots represent observations from OPTIMIZE. $E O D$ every other day, $O D$ once daily, Conc concentration prediction interval, respectively;
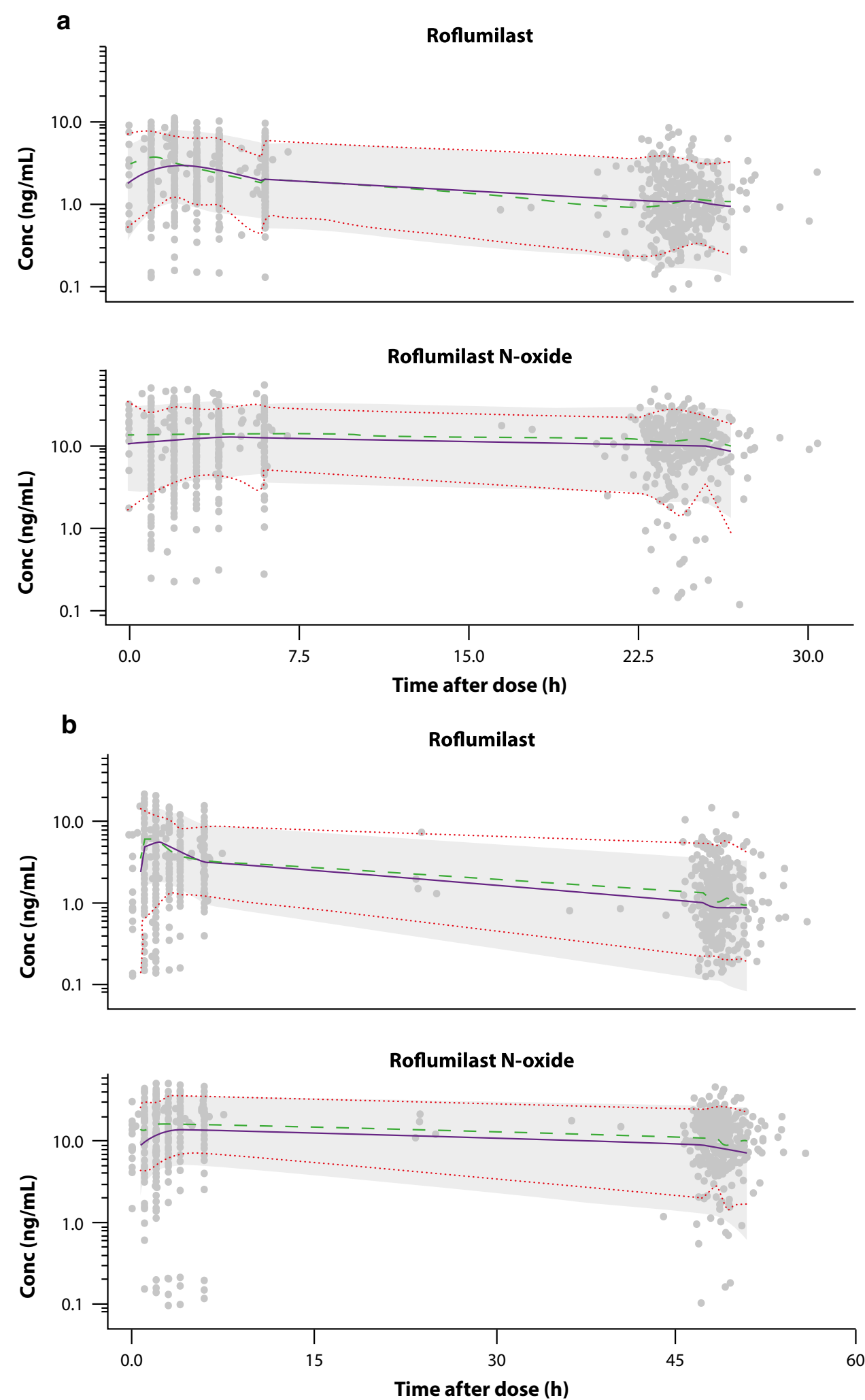

CI 0.122-0.586; $p=0.0028$ ), and higher in former smokers when compared with current smokers (effect of smoking on the logit scale: 0.357; CI 0.126-0.587; $p=0.0024)$. Treatment arm was not a significant covariate.

\subsection{PK/Time-to-Event Model}

The time-to-event model characterized the relationship between tPDE4i and time to discontinuation due to AEs. 
Fig. 2 continued
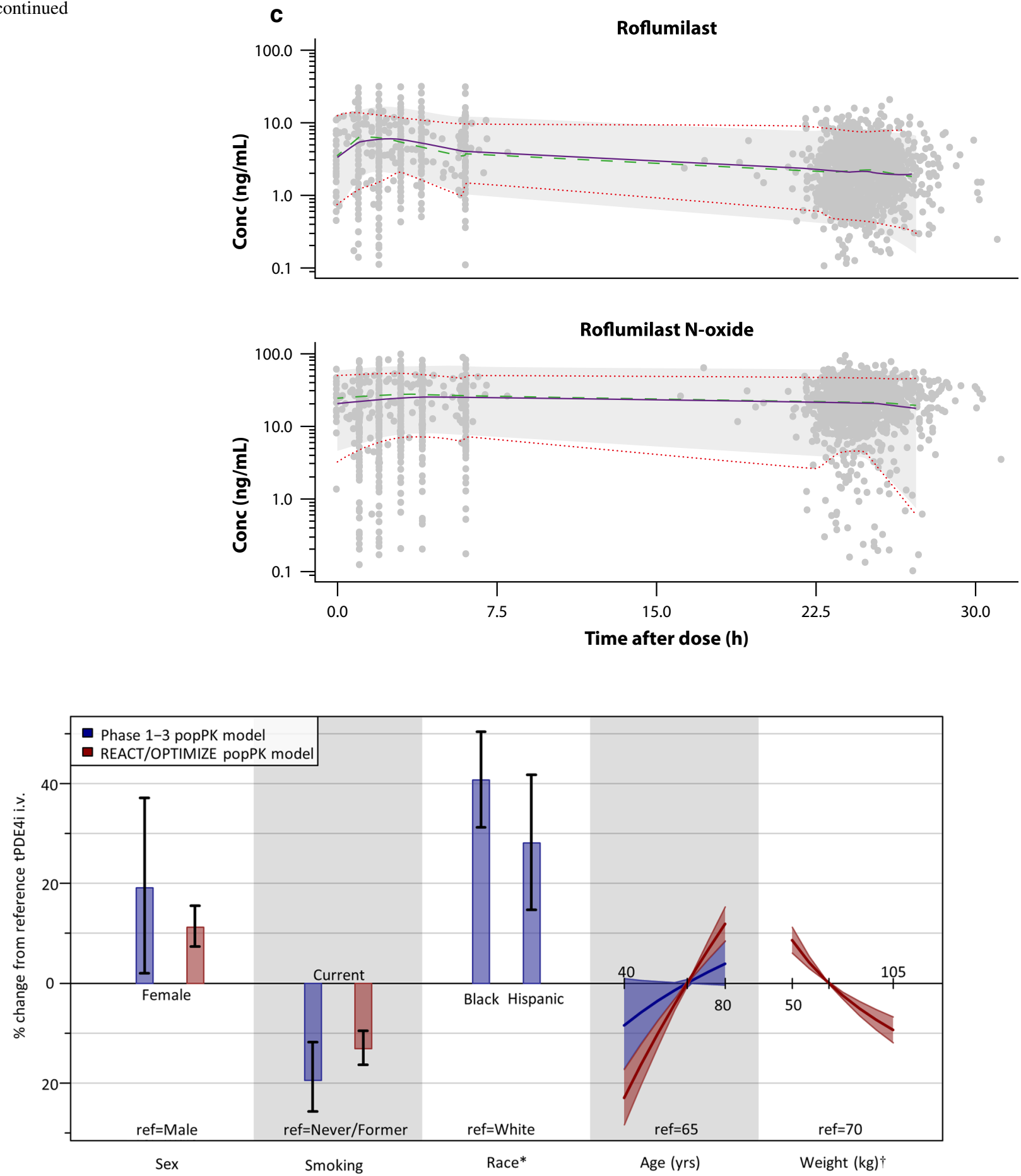

Fig. 3 Estimated covariate effects on total PDE4 inhibitory activity relative to the reference population. *Race was not tested in OPTIMIZE. †Not significant in the phase I-III popPK model. Derived covariate effects on steady state tPDE4i (red) compared with previously identified covariate effects (blue). The common

The log-normal distribution was found to best describe discontinuation event times. Therefore, the estimated position parameter corresponds to the logarithm of the geometric mean event time. reference is a male, formerly smoking, White COPD patient, aged 65 years with a body weight of $70 \mathrm{~kg}$. Data are expressed as geometric means and $68 \%$ ranges. popPK population pharmacokinetic, $t P D E 4 i$ total phosphodiesterase- 4 inhibition, i.v. intravenous, $C O P D$ chronic obstructive pulmonary disease

The PK/time-to-event model was built on up-titration data only (weeks 1-4). The final PK/time-to-event analysis predicted that patients receiving roflumilast $250 \mu \mathrm{g}$ once daily had significantly longer time to discontinuation 


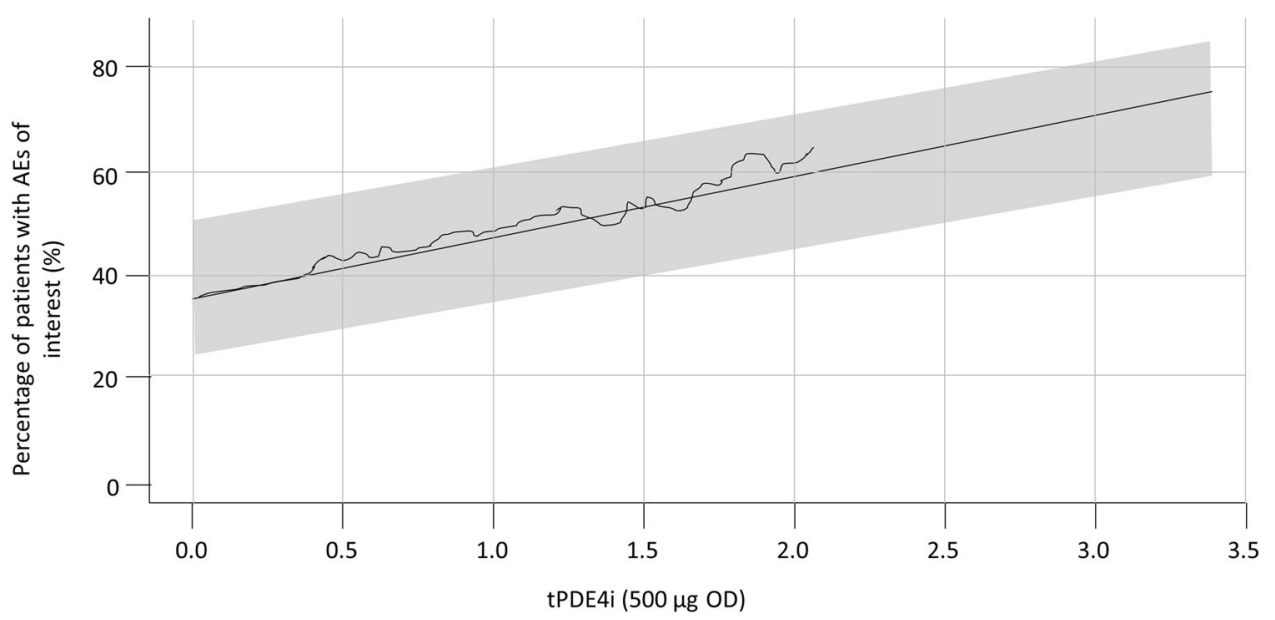

Fig. $4 \mathrm{PK} / \mathrm{AE}$ model response from the logistic regression for the percentage of patients with AEs of interest as a function of tPDE4i $(500 \mu \mathrm{g}$ OD). Univariate model response for each significant covariate. Mean model response (straight line), 95\% CIs (shaded area), locally averaged percentage of patients with AEs (curved line).

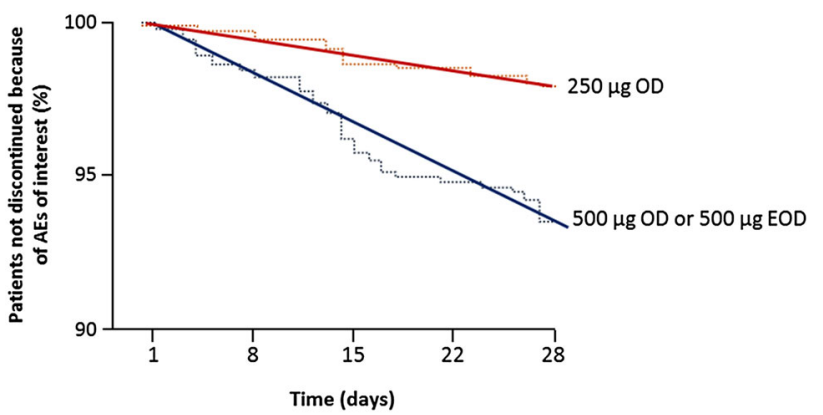

Fig. 5 PK/time-to-event model predicted typical response during the 4-week up-titration phase. Predicted percentage of patients not having discontinued due to adverse events of interest when taking roflumilast $250 \mu \mathrm{g}$ OD, $500 \mu \mathrm{g}$ OD, or $500 \mu \mathrm{g}$ EOD (straight lines). KaplanMeier estimates are also shown (dotted lines), indicating good agreement between the model and the observed events. $P K$ pharmacokinetic, $A E s$ adverse events, $O D$ once daily, $E O D$ every other day

during the up-titration phase due to AEs of interest (estimate: 1.1023; CI 0.426-1.778; $p=0.0014$ ) (Fig. 5). This estimate corresponds to a threefold $\left(\mathrm{e}^{1.1023}\right)$ increased geometric mean event time when compared with patients receiving $500 \mu \mathrm{g}$ either once daily or every other day. Furthermore, time to discontinuation was found to increase with body weight (estimate: 0.0218; CI 0.00653-0.037; $p=0.0051)$ by $2.2 \%$ per kilogram $\left(\mathrm{e}^{0.0218}\right)$.

\section{Discussion}

Parameters of the final integrated popPK model were estimated with good precision and the estimates were consistent with previous findings [14]. The model was able to adequately describe average plasma concentrations of
Note, variability is not directly related to the $95 \%$ CIs (approximately $95 \%$ of the local fits are not expected to fit within the shaded area, as with standard visual predictive check plots). $t P D E 4 i$ total phosphodiesterase- 4 inhibition, $P K$ pharmacokinetic, $A E$ adverse event, $O D$ once daily, CIs confidence intervals

roflumilast and roflumilast $\mathrm{N}$-oxide from 1238 patients, as well as BSV across study treatment phases and dosing schemes. This suggests that the existing base model previously developed was robust [12] and that the integrated popPK model accurately predicted the tPDE4i levels of roflumilast obtained from clinical data.

The predicted effects of lowering the roflumilast dose to $250 \mu \mathrm{g}$ in the first 4 weeks are consistent with the clinical findings in OPTIMIZE [15] and the earlier modelling predictions [14]. Analysis with the PK/AE model predicted that the number of patients with AEs of interest increases with roflumilast exposure. Analysis using the PK/time-toevent model predicted that patients receiving the $250 \mu \mathrm{g}$ up-titration regimen have a significantly longer time to discontinuation due to AEs of interest versus other patients treated with roflumilast $500 \mu \mathrm{g}$ every other day or $500 \mu \mathrm{g}$ once daily.

The percentage of patients predicted to have AEs of interest was increased in certain subpopulations, namely in patients concomitantly treated with LAMAs and in former smokers. However, the covariate effects were small compared with the overall variability, such that the risk ratios were similar across all simulated subpopulations. Furthermore, there are no known pharmacological reasons why concomitant treatment with a LAMA should increase sensitivity to inhibition of PDE4 [18, 19], although downstream effects of modulating cyclic adenosine monophosphate (cAMP) levels cannot be excluded [20].

As in our previous popPK analysis [12], we identified smoking as being a significant covariate in the disposition of roflumilast and roflumilast $\mathrm{N}$-oxide. Roflumilast is converted to roflumilast $\mathrm{N}$-oxide by cytochrome $\mathrm{P} 450$ (CYP) 3A4 and 1A2 isoenzymes [12]. Smoking is a known 
inducer of CYP1A2 and would reflect in higher clearance and consequently lower exposure of roflumilast for smokers [21]. Our analysis showed that patients with lower exposure have a lower probability of developing a treatment-emergent $\mathrm{AE}$, which was consistent with our previous experience regarding the relationship between exposure and the probability of AEs, and also confirms the impact of smoke-induced CYP1A2 in the disposition of roflumilast. Although the effect was small, smoking status was also identified as a covariate in the PK/AE model, and we found that smokers had less AEs of interest compared with former smokers, including with placebo, and therefore may reflect a difference in the health status of these subjects as opposed to a difference in susceptibility to roflumilast treatment.

Interestingly, the time-to-event analysis found that the time to discontinuation due to AEs increased with body weight, suggesting that patients with high body weight may be better able to manage AEs such as diarrhoea and nausea and continue roflumilast treatment.

Limitations of this analysis include the lack of characterization of discontinuation after the initial 4-week treatment period in the time-to-event analysis (as exposure is not independent of time and the dose changes after week 4). In addition, the PK/AE logistic regression model does not have a time component; therefore, only one summary value was used per patient to characterize exposure.

\section{Conclusions}

The integrated popPK model accurately predicted the tPDE4i levels obtained from clinical study data. PK/AE and $\mathrm{PK} /$ time-to-event models indicate that discontinuation events due to AEs are significantly reduced and onset is delayed in patients up-titrated with $250 \mu \mathrm{g}$ once daily for 4 weeks before continuing with $500 \mu \mathrm{g}$ once daily, compared with patients starting treatment with $500 \mu \mathrm{g}$ once daily or every other day. The lower dose may not induce sufficient PDE4 inhibition to exert clinical efficacy, and hence is not recommended as long-term maintenance therapy. An initial 4-week up-titration regimen of roflumilast at a dose of $250 \mu \mathrm{g}$ daily can reduce the risk of side effects and early treatment discontinuations associated with roflumilast, thereby helping patients to continue to receive the effective maintenance daily dose of $500 \mu \mathrm{g}$.

Acknowledgements Axel Facius and Gezim Lahu were formerly employed by Takeda Pharmaceuticals International GmbH, Zurich, Switzerland. The authors thank Anne Keunecke, Jean Smeets and Nelleke Snelder for the development and interpretation of the phase I-III, REACT popPK and PK/PD models; Teun Post and Maurice Ahsman (all employed by LAP\&P Consultants B.V., Leiden, The Netherlands) for the development and interpretation of the popPK and
$\mathrm{PK} / \mathrm{FEV}_{1}$ models for OPTIMIZE; and Ella Palmer, $\mathrm{PhD}$, an employee of Synergy Vision, London, UK, for providing medical writing support, funded by AstraZeneca, in accordance with Good Publication Practice (GPP3) guidelines (http://www.ismpp.org/gpp3).

\section{Compliance with Ethical Standards}

Funding This study was funded by Takeda Pharmaceuticals International GmbH, Zurich, Switzerland; AstraZeneca, Cambridge, UK, are the current study sponsors.

Conflict of interest Henrik Watz has received consulting fees and payment for lectures from Takeda and AstraZeneca. He has received support for travel to meetings for the study and manuscript preparation from AstraZeneca, and provided writing assistance to AstraZeneca. Philip Gardiner is an employee of AstraZeneca. Axel Facius was employed as pharmacometrician at Takeda Pharmaceuticals, who executed the studies reported in this manuscript. He further received travel support from AstraZeneca for the ATS2017 conference, where parts of the current manuscript were presented. Gezim Lahu was employed by Takeda, who executed the studies reported in this manuscript. Eleonora Marostica was a paid consultant for Takeda.

Ethical approval All procedures performed in studies involving human participants were in accordance with the ethical standards of the institutional and/or national research committee and with the 1964 Helsinki declaration and its later amendments or comparable ethical standards.

Informed consent Informed consent was obtained from all individual participants included in the study. Additional informed consent was obtained from all individual participants for whom identifying information is included in this article.

Open Access This article is distributed under the terms of the Creative Commons Attribution-NonCommercial 4.0 International License (http://creativecommons.org/licenses/by-nc/4.0/), which permits any noncommercial use, distribution, and reproduction in any medium, provided you give appropriate credit to the original author(s) and the source, provide a link to the Creative Commons license, and indicate if changes were made.

\section{References}

1. The Global Initiative for Chronic Obstructive Lung Disease (GOLD) Global Strategy for the Diagnosis, Management, and Prevention of Chronic Obstructive Pulmonary Disease. 2017. www.goldcopd.org. Accessed 12 May 2017.

2. Stockley RA. Progression of chronic obstructive pulmonary disease: impact of inflammation, comorbidities and therapeutic intervention. Curr Med Res Opin. 2009;25(5):1235-45.

3. Wedzicha JA, Seemungal TA. COPD exacerbations: defining their cause and prevention. Lancet. 2007;370(9589):786-96.

4. Hurst JR, Vestbo J, Anzueto A, Locantore N, Mullerova H, TalSinger R, et al. Susceptibility to exacerbation in chronic obstructive pulmonary disease. N Engl J Med. 2010;363(12):1128-38.

5. Wedzicha JA, Calverley PM, Rabe KF. Roflumilast: a review of its use in the treatment of COPD. Int $\mathrm{J}$ Chron Obstruct Pulmon Dis. 2016;11:81-90.

6. Bateman ED, Rabe KF, Calverley PM, Goehring UM, Brose M, Bredenbroker D, et al. Roflumilast with long-acting \{beta\}2 
agonists for COPD: influence of exacerbation history. Eur Respir J. 2011;38(3):553-60.

7. Calverley PM, Rabe KF, Goehring UM, Kristiansen S, Fabbri LM, Martinez FJ. Roflumilast in symptomatic chronic obstructive pulmonary disease: two randomised clinical trials. Lancet. 2009;374(9691):685-94.

8. Martinez FJ, Calverley PM, Goehring UM, Brose M, Fabbri LM, Rabe KF. Effect of roflumilast on exacerbations in patients with severe chronic obstructive pulmonary disease uncontrolled by combination therapy (REACT): a multicentre randomised controlled trial. Lancet. 2015;385(9971):857-66.

9. DAXAS $500 \mu \mathrm{g}$ tablets-summary of product characteristics (SPC). 2015. Last updated 24 Apr 2017. http://www.ema.europa. eu/docs/en_GB/document_library/EPAR_-_Product_ Information/human/001179/WC500095209.pdf. Accessed 30 Mar 2017.

10. Calverley PM, Martinez FJ, Fabbri LM, Goehring UM, Rabe KF. Does roflumilast decrease exacerbations in severe COPD patients not controlled by inhaled combination therapy? The REACT study protocol. Int J Chron Obstruct Pulmon Dis. 2012;7:375-82.

11. Hatzelmann A, Morcillo EJ, Lungarella G, Adnot S, Sanjar S, Beume R, et al. The preclinical pharmacology of roflumilast-a selective, oral phosphodiesterase 4 inhibitor in development for chronic obstructive pulmonary disease. Pulm Pharmacol Ther. 2010;23(4):235-56.

12. Lahu G, Hunnemeyer A, Diletti E, Elmlinger M, Ruth P, Zech K, et al. Population pharmacokinetic modelling of roflumilast and roflumilast $\mathrm{N}$-oxide by total phosphodiesterase- 4 inhibitory activity and development of a population pharmacodynamic-adverse event model. Clin Pharmacokinet. 2010;49(9):589-606.

13. Lahu G, Nassr N, Hunnemeyer A. Pharmacokinetic evaluation of roflumilast. Expert Opin Drug Metab Toxicol. 2011;7(12):1577-91.

14. Lahu G, Facius A. Application of population pharmacokinetic modeling to explore the impact of alternative roflumilast dosing regimens on tolerability. Int $\mathbf{J}$ Clin Pharmacol Ther. 2013;51(11):832-6.

15. Watz H, Bagul N, Rabe KF, Rennard S, Alagappan VKT, Román $\mathrm{J}$, et al. Use of a 4-week up-titration regimen of roflumilast in patients with severe COPD. Int J Chron Obstruct Pulmon Dis. 2018;13:813-22.

16. Schwarz G. Estimating the dimension of a model. Ann Stat. 1978;6:461-4.

17. Jonsson EN, Karlsson MO. Automated covariate model building within NONMEM. Pharm Res. 1998;15(9):1463-8.

18. Houslay MD, Baillie GS, Maurice DH. cAMP-Specific phosphodiesterase-4 enzymes in the cardiovascular system: a molecular toolbox for generating compartmentalized cAMP signaling. Circ Res. 2007;100(7):950-66.

19. Matera MG, Cazzola M. Muscarinic receptor antagonists. Handb Exp Pharmacol. 2017;237:41-62.

20. Spina D. PDE4 inhibitors: current status. Br J Pharmacol. 2008;155(3):308-15.

21. Funck-Brentano C, Raphael M, Lafontaine M, Arnould JP, Verstuyft C, Lebot M, et al. Effects of type of smoking (pipe, cigars or cigarettes) on biological indices of tobacco exposure and toxicity. Lung Cancer. 2006;54(1):11-8. 